\title{
Sex-related and racial variations in orbital floor anatomy
}

\section{Seung Jin Moon, \\ Won Jai Lee, \\ Tai Suk Roh, Wooyeol Baek}

Department of Plastic and

Reconstructive Surgery, Institute for

Human Tissue Restoration, Severance

Hospital, Yonsei University College of

Medicine, Seoul, Korea

\begin{abstract}
Background: Repair of the orbital floor following trauma or tumor removal remains a challenge because of its complex three-dimensional shape. The purpose of the present study is to understand normal orbital floor anatomy by investigating its differences across four groups (Caucasian American and East Asian, males and females) via facial bone computed tomography (CT).

Methods: A total of 48 orbits in 24 patients between 20 and 60 years of age were evaluated. Although most patients underwent CT scanning following trauma, the orbital walls were intact in all patients. Linear and angular measurements of the orbital floor were obtained from CT images. Results: Orbital floor width, length, angle between the orbital floor and medial wall, and distance from the inferior orbital rim to the lowest point of the orbital floor did not show a statistically significant difference between groups. Angles made by the infraorbital rim, the lowest point of the floor, and the anterior border of the infraorbital fissure were statistically significantly wider in East Asian females than in male groups. The floor depth in East Asian females was significantly smaller compared to all the other groups.

Conclusion: East Asian female population had smaller curvature and depth of an orbital floor than the other groups, which means racial and sex-related differences should be considered in the orbital floor reconstruction.
\end{abstract}

Keywords: Anatomy / Orbit / Race factor / Sex factor

\section{INTRODUCTION}

The involvement of the orbital structure in craniofacial trauma is not unusual, and its rate was reported up to $40 \%$ [1]. In most of these cases, there are major changes to the anatomy of the orbital floor, and it may contribute to an increase in the volume of the orbit [2]. Medial wall fractures often occur with fractures of the orbital floor, particularly in the transition point between the two bony structures. Such fractures may lead to restricted horizontal and vertical movements of the eyeball. Accurate reconstruction and reposition of the injured orbital anatomy be-

Correspondence: Wooyeol Baek

Department of Plastic and Reconstructive Surgery, Yonsei University College of

Medicine, 50-1 Yonsei-ro, Seodaemun-gu, Seoul 03722, Korea

E-mail: parande@yuhs.ac

Received April 17, 2020 / Revised May 31, 2020 / Accepted June 1, 2020 comes critical in such cases as post-traumatic deformities could lead to severe complications (e.g., loss of visual acuity, vertical strabismus, and diplopia). Enophthalmos and loss of sensory perception on area innervated by the infraorbital nerve also can be presented as sequelae [3].

Due to advances in surgical techniques and diagnostic imaging, computed tomography (CT) is now the gold standard for precise three-dimensional (3D) anatomical measurement and planning required for orbital reconstructions. CT imaging has been used to create anatomical models that serve as templates for bone grafts and 3D titanium mesh implants [4,5]. However, due to the complex structure of the orbit, with its characteristic S-shaped craniocaudal rise in the inferior wall and retrobulbar bulge formed by the medial wall, accurate reconstruction of the orbital floor remains a surgical challenge [6]. 
There have been attempts to understand normal orbital anatomy through CT, but measurements in most of those studies had been made under the assumption that the orbital wall is flat $[7,8]$. Furthermore, no studies have assessed racial variation in these measures. Thus, the present study assessed sex-related and racial variations in the orbital floor anatomy to contribute to knowledge on variations in the 3D morphology of the orbital floor.

\section{METHODS}

A retrospective review including patients, ranging from 20 to 60 years of age, who had visited Yonsei University Severance Hospital and undergone facial CT scans between January 2017 and December 2019 was performed. Twelve East Asian (EA) and 12 Caucasian American (CA) adults (six men and six women in each group) were randomly selected for the study and patients with any abnormality of the orbit were excluded. GE Centricity PACS software (GE Healthcare, Chicago, IL, USA) was used to analyze all CT images.

To evaluate the size of the orbital floor, we measured its width and length. A coronal plane was selected at the level of the lateral orbital rim, and a horizontal line passing through the ethmoidomaxillary suture was made. The orbital floor width was defined as the length of that line as it passed within the orbital cavity (Fig. 1). The angle formed by the ethmoid bone's lamina papyracea, the ethmoidomaxillary suture, and the infraorbital groove on the same coronal plane was used to determine the angular relationship between the medial wall and floor and termed the anterior inferomedial angle (Fig. 2). Although the orbital floor is a triangular shape with the optic canal as an apex, fracture occurs mainly in a large area near the base. Therefore, to determine the size of the field actually considered during reconstruction, we defined the orbital floor length as the distance between the inferior orbital rim and the inferior orbital fissure on a sagittal plane bisecting the line of the orbital floor width (Fig. 3).

To analyze the curvature of the orbital floor, some measurements were taken on the sagittal plane passing the lowest point of the orbital floor (Fig. 4). The sagittal angle was defined as the angle made by the infraorbital rim, the lowest point of the floor, and the anterior border of the infraorbital fissure. The floor depth' was the distance between the lowest point of the orbital floor and the line connecting the infraorbital rim and the anterior border of the inferior orbital fissure. The "sagittal distance from the inferior orbital rim to the lowest point (SDIL)" was

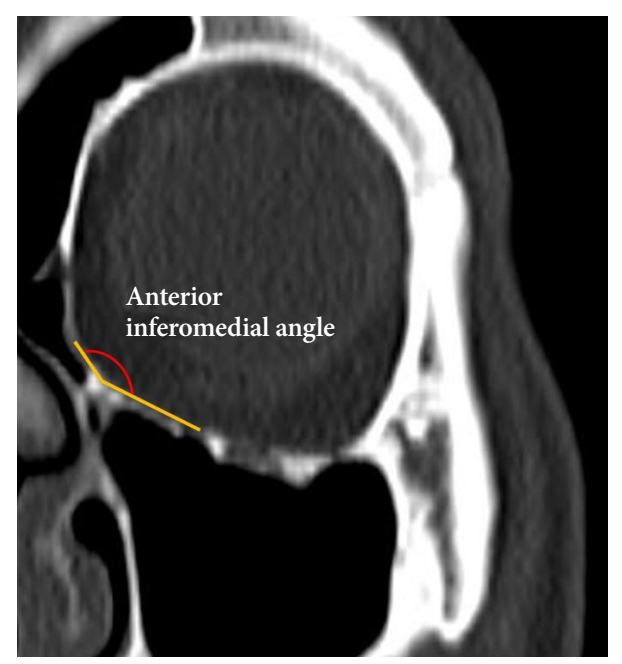

Fig. 2. Definition of the anterior inferomedial angle.

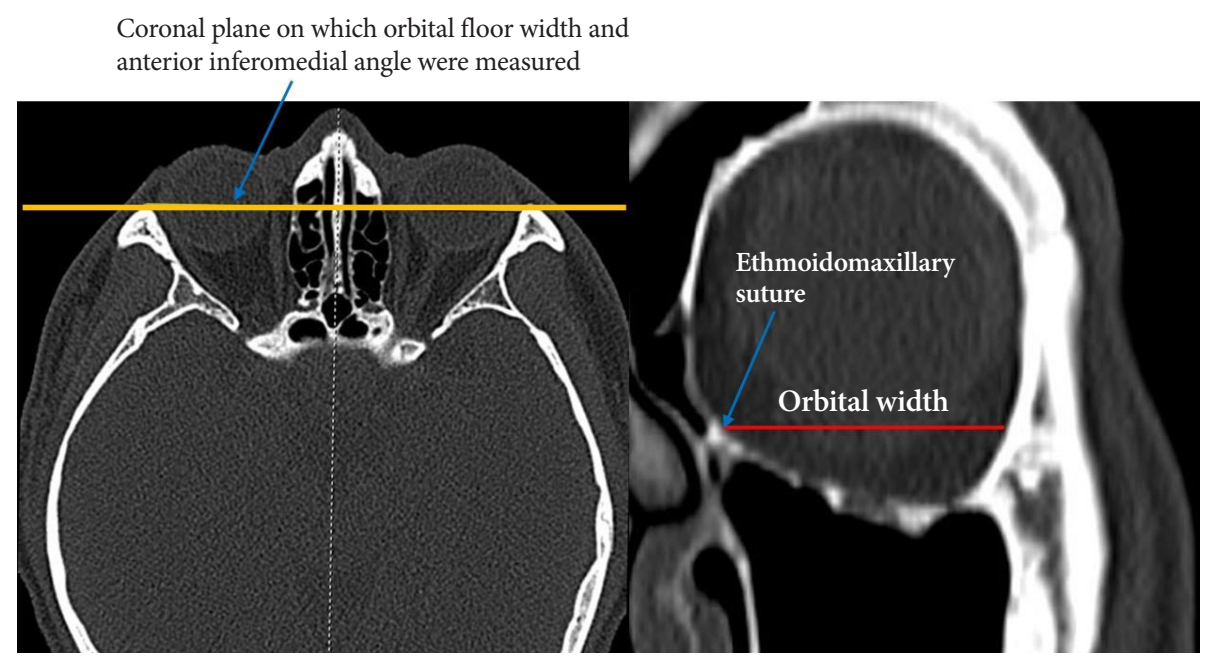

Fig. 1. Selection of coronal planes on which orbital floor widths and anterior inferomedial angles were measured and definition of the orbital floor width. 


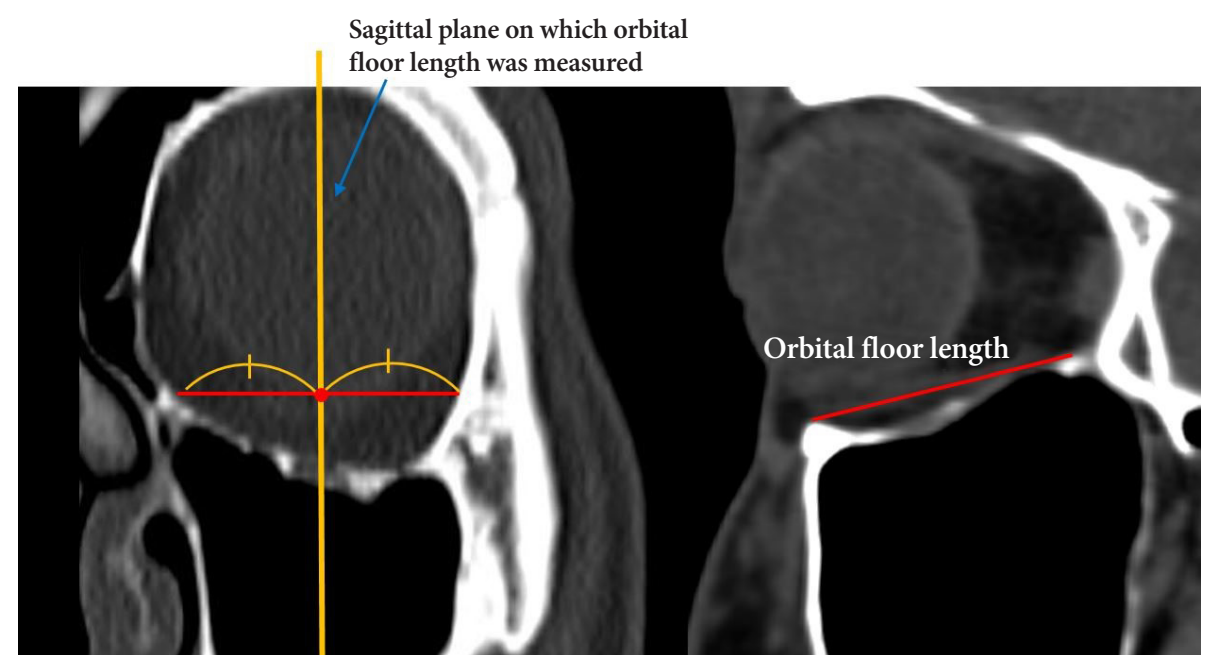

Fig. 3. Selection of sagittal planes on which orbital floor lengths were measured and definition of the orbital floor length.

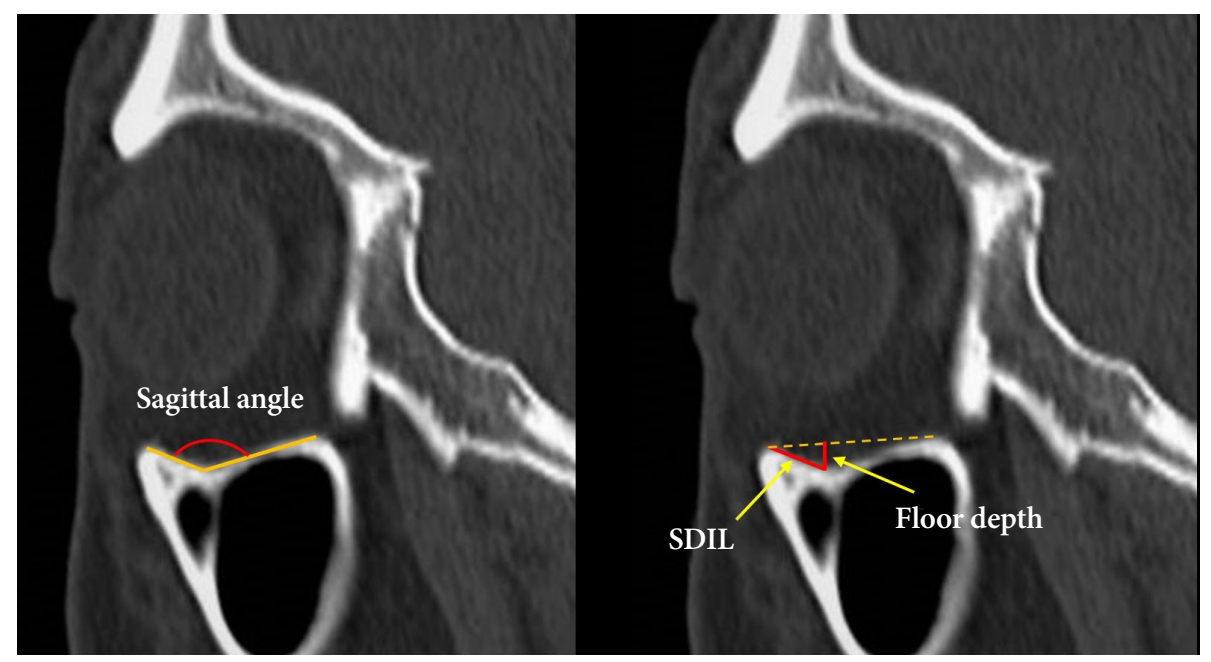

Fig. 4. Definition of the sagittal angle, the sagittal distance from the inferior orbital rim to the lowest point (SDIL), and the floor depth measured on the sagittal plane.

defined as the distance between the infraorbital rim and the lowest point of the orbital floor.

Data were analyzed using the Statistical Package for Social Sciences (SPSS) version 23.0 (IBM Corp., Armonk, NY, USA). In each patient, both right and left orbital floors were measured, and the average values were used for the analysis. While each measurement was similar to a normal distribution according to the normality test (Kolmogorov-Smirnov, Shapiro-Wilk test), each of the four groups were compared by F-tests of one-way analyses of variance (ANOVA) and Scheffe comparison. A posthoc ANOVA power analysis to determine if there was sufficient sample size to detect a statistical difference was performed using $G^{\star}$ Power software version 3.1 (Dusseldorf, Germany). T-tests for mean difference were used when comparing any two groups. Statistical significance was determined as $p<0.05$.

\section{RESULTS}

Based on sex and race, the 24 patients were divided into four groups. There was no statistically significant age difference between groups. The mean orbital floor width of all the patients was $35.48 \pm 1.85 \mathrm{~mm}$, and the orbital floor length was $27.69 \pm$ $2.44 \mathrm{~mm}$. The width and length of the orbital floor did not statistically significantly different between groups, indicating that sex or race did not influence the size of the orbital floor (Figs. 5, 6). The anterior inferomedial angle was the largest among $\mathrm{CA}$ males $\left(140.6^{\circ} \pm 7.0^{\circ}\right)$, followed by CA females $\left(138.1^{\circ} \pm 10.5^{\circ}\right)$, EA males $\left(132.4^{\circ} \pm 8.4^{\circ}\right)$, and EA females $\left(129.3^{\circ} \pm 14.2^{\circ}\right.$ ) (Fig. 7). However, there was no statistically significant difference between the groups. The average anterior inferomedial angle of all the subjects was $135.1^{\circ} \pm 10.7^{\circ}$. 


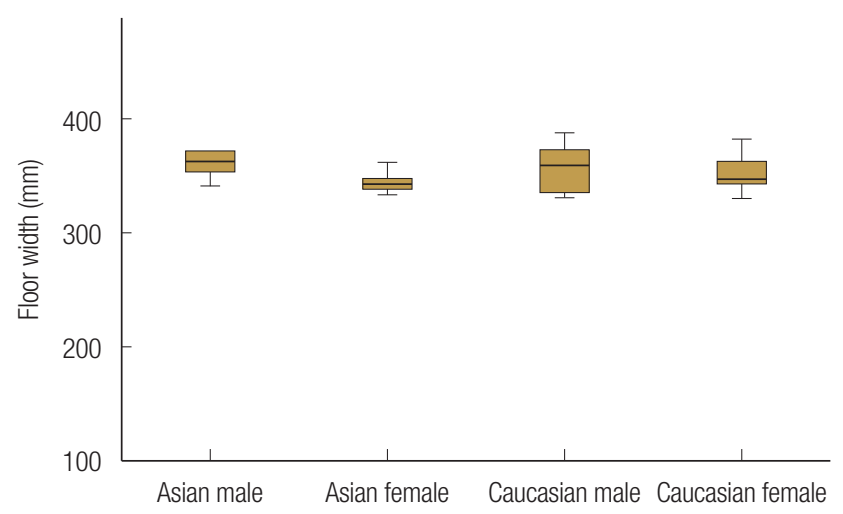

Fig. 5. Sex-related and racial variation in the orbital floor width.

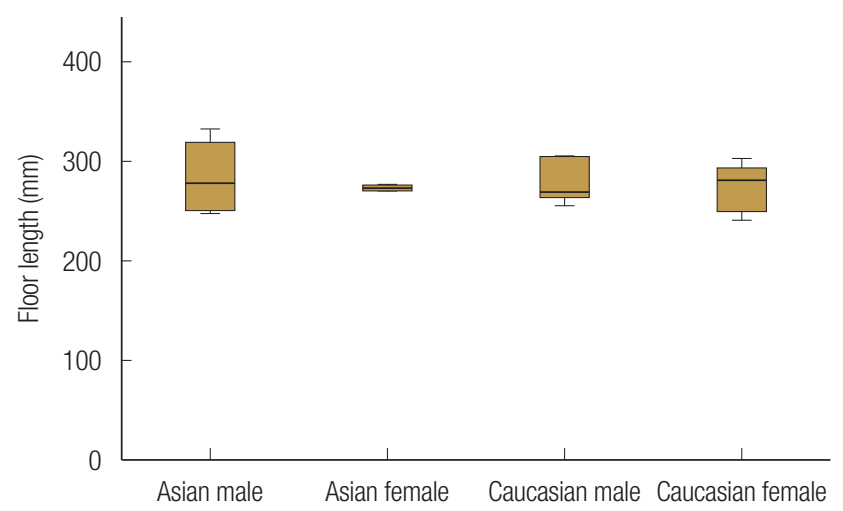

Fig. 6. Sex-related and racial variation in the orbital floor length.

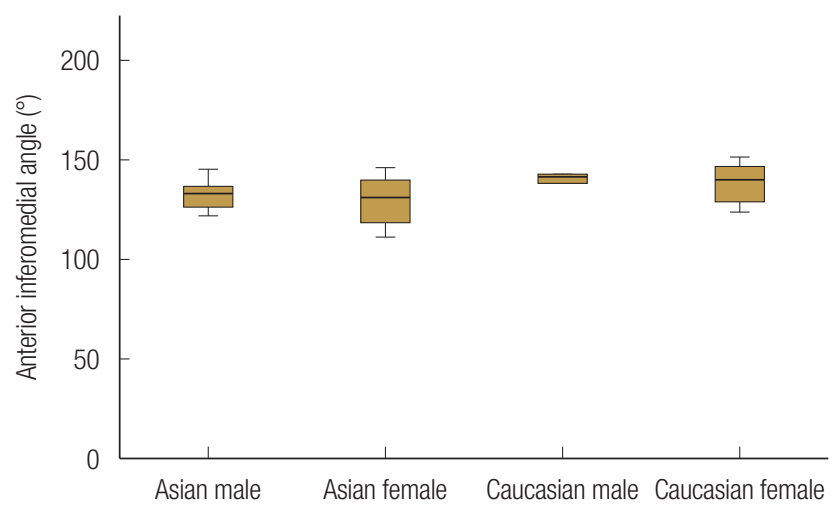

Fig. 7. Sex-related and racial variation in the anterior inferomedial angle.

EA females $\left(167.6^{\circ} \pm 5.0^{\circ}\right)$ had a statistically significantly wider sagittal angle compared with both male groups (EA males: $146.4^{\circ} \pm 8.9^{\circ}$, CA males: $\left.154.6^{\circ} \pm 7.7^{\circ}, p<0.05\right)$. The sagittal angle of CA females $\left(158.3^{\circ} \pm 3.3^{\circ}\right)$ showed statistically significantly wider than EA ( $p=0.045)$ (Fig. 8). The average value of the SDIL was $5.80 \pm 1.14 \mathrm{~mm}$, and the mean floor depth was $3.10 \pm 1.31$ $\mathrm{mm}$. The SDIL did not statistically significantly different between groups (Fig. 9). Floor depths were similar across the three

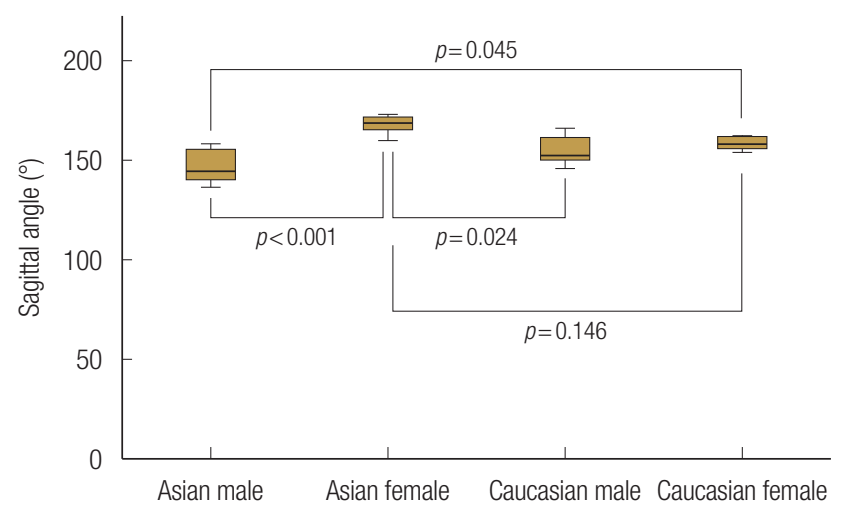

Fig. 8. Effect of sex and race on the sagittal angle.

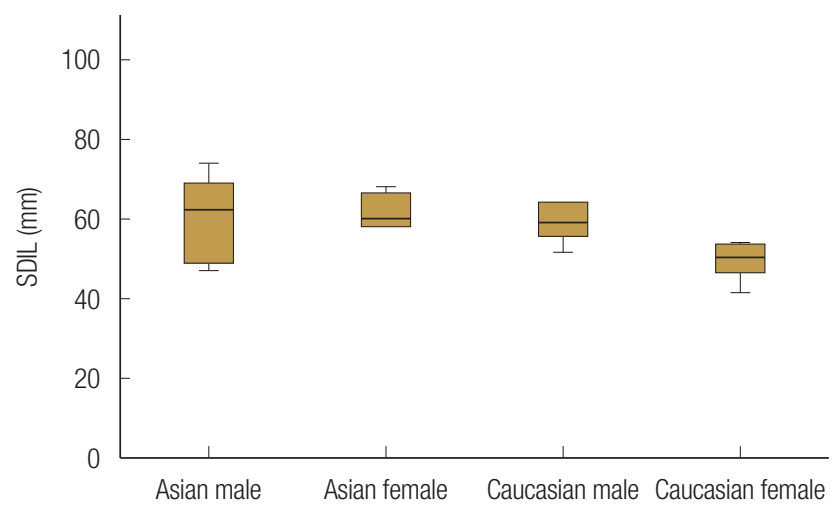

Fig. 9. Effect of sex and race on the sagittal distance from the inferior orbital rim to the lowest point (SDIL).

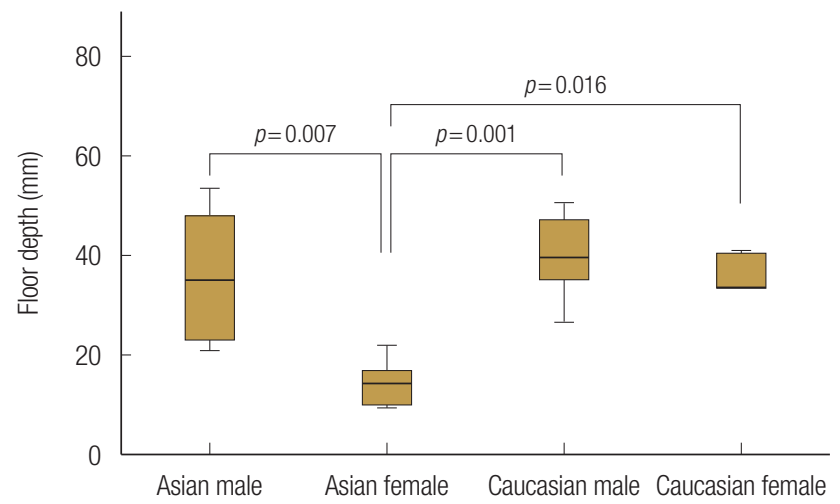

Fig. 10. Effect of sex and race on the floor depth.

groups (Asian males: $3.60 \pm 1.35 \mathrm{~mm}$; CA males: $3.97 \pm 8.93$ $\mathrm{mm}$; CA females: $3.10 \pm 7.40 \mathrm{~mm}$ ). However, EA females had significantly smaller floor depth compared with the other groups $(1.45 \pm 4.71 \mathrm{~mm}, p<0.05)$ (Fig. 10). Post-hoc power analysis for sagittal angle and floor depth demonstrated that the sample size was sufficient to determine statistical differences.

To find factors contributing to the sagittal angle and the floor depth, sex-related and racial differences were separately ana- 
lyzed. There was no significant difference in sagittal angle between EA and CA (EA: $157.0^{\circ} \pm 13.0^{\circ}, \mathrm{CA}: 156.4^{\circ} \pm 6.0^{\circ}, p=$ 0.889 ), but sagittal angle of the female participants showed statistically significantly wider than the males (male: $150.5^{\circ} \pm 9.0^{\circ}$, female: $162.9^{\circ} \pm 6.3^{\circ}, p=0.001$ ). Floor depths were statistically significantly smaller in the EA population than in the CA population (EA: $25.2 \pm 14.7 \mathrm{~mm}, \mathrm{CA}: 36.7 \pm 8.4 \mathrm{~mm}, p=0.028$ ). Floor depths also showed a statistically significant difference between the males and the females (male: $37.8 \pm 11.1 \mathrm{~mm}$, female: $24.1 \pm 11.7 \mathrm{~mm}, p=0.007)$.

\section{DISCUSSION}

The orbital floor is the most common isolated orbital bone fracture site in head trauma cases, and zygomaticomaxillary fractures often accompany fractures in the orbital floor $[3,9]$. However, the complex 3D structure of the orbital cavity and the narrow operating field render it difficult to repair orbital wall defects accurately. Incorrect reconstruction can lead to diplopia, a limit of motion of eye movement, enophthalmos, or exophthalmos. However, advances in technologies such as virtual planning and 3D printing have led to the development of more accurate and innovative orbital wall reconstruction techniques. For example, orbital floor reconstruction using virtual reconstruction software and 3D printed facial bone model showed effective and satisfactory results [5,10]. Maxillofacial, otorhinolaryngological, and plastic surgeons had successfully worked with other modeling methods such as the computer-assisted navigation systems where the mirror image of the intact contralateral orbit is employed to reconstruct the orbital floor [11]. However, while these new techniques offer great clinical promise, they are not commonly available at all institutions due to their cost and manufacturing time issues. Given the limitations mentioned, a surgeon's understanding and experience with normal orbital floor anatomy are essential for successful reconstruction. Some previous studies about orbital anatomy showed that the morphology of the orbit is heterogeneous and is potentially affected by sex, race, and age $[12,13]$. In this study, we focused on analyzing anatomical variations by sex and race of the orbital floor in EA and CA populations.

Although the orbital floor is the single most fractured site among orbital walls, blow-out fractures involving both the medial wall and the orbital floor are also common [3,14]. For reconstructing the medial and inferior walls together, angular relationships between the two walls are critical factors. Analysis of the Anterior inferomedial angle demonstrated that the orbital floor and medial wall met at an average angle of about $135^{\circ}$, regardless of race or sex. This finding could be useful when re- constructing orbital floor and medial wall together.

The mean sagittal angle, which is related with a curvature of an orbital floor in sagittal direction, was $156.7^{\circ}$. Notably, we found that the sagittal angle was significantly higher in EA females, indicating that their orbital floors were flatter than other populations. This finding suggests that surgeons who are planning to reconstruct an orbital floor should give attention especially to the variation of the curvature. Unlike the comparison between the two sex groups, the sagittal angle did not show a statistically significant difference between the two racial groups. Furthermore, the floor depth showed a greater difference between men and women than between the races. This finding means that orbital floor anatomy was more affected by sex than by race.

Research on orbital floor anatomy by Nagasao et al. [12] (including 182 patients at Keio University Hospital) revealed that the location of the lowest point moves postero-inferiorly with increasing age, with no significant difference between male and female participants. However, in our study, there were statistically significant differences in some measurements between the males and the females, and we did not analyze age factor because of the small sample size. For further and accurate analysis of factors contributing to orbital floor anatomy, increasing the sample size across multiple ages will be needed in the future study.

To the best of our knowledge, this is the first study investigating the racial and sex-related variation of orbital floor in the CA and EA populations. The length and width of the orbital floor and relationship between the medial and inferior walls were similar regardless of sex or race. However, orbital floor curvature in the sagittal direction differed significantly between groups and was almost flat in EA females. This quantitative analysis of the $3 \mathrm{D}$ shape of the orbital floor could be helpful for a successful reconstruction of an orbital floor.

\section{NOTES}

\section{Conflict of interest}

No potential conflict of interest relevant to this article was reported.

\section{Ethical approval}

The study was approved by the Institutional Review Board of Severance Hospital, Yonsei University Health System (IRB No. 4-2020-0213) and performed in accordance with the principles of the Declaration of Helsinki. Written informed consents were obtained. 


\section{ORCID}

Seung Jin Moon

https://orcid.org/0000-0003-1980-0270

Won Jai Lee

Tai Suk Roh

Wooyeol Baek
https://orcid.org/0000-0001-8681-159X

https://orcid.org/0000-0002-6638-4110

\section{REFERENCES}

1. Hoffmann J, Cornelius CP, Groten M, Probster L, Pfannenberg C, Schwenzer N. Orbital reconstruction with individually copy-milled ceramic implants. Plast Reconstr Surg 1998;101:60412.

2. Chang EW, Manolidis S. Orbital floor fracture management. Facial Plast Surg 2005;21:207-13.

3. Manolidis S, Weeks BH, Kirby M, Scarlett M, Hollier L. Classification and surgical management of orbital fractures: experience with 111 orbital reconstructions. J Craniofac Surg 2002; 13:726-37.

4. Kozakiewicz M, Elgalal M, Loba P, Komunski P, Arkuszewski P, Broniarczyk-Loba A, et al. Clinical application of 3D pre-bent titanium implants for orbital floor fractures. J Craniomaxillofac Surg 2009;37:229-34.

5. Vehmeijer M, van Eijnatten M, Liberton N, Wolff J. A novel method of orbital floor reconstruction using virtual planning, 3-dimensional printing, and autologous bone. J Oral Maxillofac Surg 2016;74:1608-12.

6. Hammer B, Prein J. Correction of post-traumatic orbital defor-

mities: operative techniques and review of 26 patients. J Craniomaxillofac Surg 1995;23:81-90.

7. Kang HS, Han JJ, Oh HK, Kook MS, Jung S, Park HJ. Anatomical studies of the orbital cavity using three-dimensional computed tomography. J Craniofac Surg 2016;27:1583-8.

8. Weaver AA, Loftis KL, Tan JC, Duma SM, Stitzel JD. CT based three-dimensional measurement of orbit and eye anthropometry. Invest Ophthalmol Vis Sci 2010;51:4892-7.

9. Hwang K, You SH, Sohn IA. Analysis of orbital bone fractures: a 12-year study of 391 patients. J Craniofac Surg 2009;20:121823.

10. Kim YC, Jeong WS, Park TK, Choi JW, Koh KS, Oh TS. The accuracy of patient specific implant prebented with 3D-printed rapid prototype model for orbital wall reconstruction. J Craniomaxillofac Surg 2017;45:928-36.

11. Schmelzeisen R, Gellrich NC, Schoen R, Gutwald R, Zizelmann C, Schramm A. Navigation-aided reconstruction of medial orbital wall and floor contour in cranio-maxillofacial reconstruction. Injury 2004;35:955-62.

12. Nagasao T, Hikosaka M, Morotomi T, Nagasao M, Ogawa K, Nakajima T. Analysis of the orbital floor morphology. J Craniomaxillofac Surg 2007;35:112-9.

13. Kumaran A, Chan A, Yong K, Shen S. Ethnic variation in deep lateral orbital anatomy and its implications on decompression surgery. Orbit 2019;38:95-102.

14. Patnaik VVG, Bala S, Singla R. Anatomy of the bony orbitsome applied aspects. J Anat Soc India 2001;50:59-67. 